\title{
Regards croisés sur l'introduction du discours direct en anglais et en français : mise en contexte dans The Pearl et La perle de John Steinbeck
}

\section{Grégoire Lacaze}

\section{(2) OpenEdition \\ Journals \\ Édition électronique \\ URL : http://journals.openedition.org/esa/1625 \\ DOI : 10.4000/esa. 1625 \\ ISSN : 2650-2623 \\ Éditeur \\ Société de stylistique anglaise}

Édition imprimée

Date de publication : 31 décembre 2011

Pagination : 89-106

ISSN : 2116-1747

\section{Référence électronique}

Grégoire Lacaze, «Regards croisés sur l'introduction du discours direct en anglais et en français mise en contexte dans The Pearl et La perle de John Steinbeck », Études de stylistique anglaise [En ligne], 3 | 2011, mis en ligne le 27 novembre 2018, consulté le 19 avril 2019. URL : http://

journals.openedition.org/esa/1625; DOI : 10.4000/esa.1625 


\title{
REGARDS CROISÉS SUR L'INTRODUCTION DU DISCOURS DIRECT EN ANGLAIS ET EN FRANÇAIS : MISE EN CONTEXTE DANS THE PEARL ET LA PERLE DE JOHN STEINBECK
}

\author{
LACAZE Grégoire \\ Aix-Marseille Université - LERMA - EA 853
}

\begin{abstract}
:
This paper focuses on the introduction of direct speech in works of fiction originally written in English and in their translations into French. The contrastive approach adopted in this study aims at comparing the use of direct speech in Steinbeck's The Pearl and its translation into French, La perle. Syntactic, semantic and stylistic notions are used to highlight and analyze the strategies chosen by the French translators Marcel Duhamel and Renée Vavasseur.

\section{Résumé :}

Nous nous proposons d'étudier l'introduction du discours direct dans des œuvres de fiction en anglais et dans leur traduction en français. L'analyse contrastive qui sera menée dans cette étude comparera les stratégies mises en œuvre pour introduire du discours direct dans The Pearl de John Steinbeck et dans sa traduction en français, La perle. Cette analyse tentera de mettre en évidence les choix auxquels sont confrontés les traducteurs en fonction des contraintes imposées par le texte source. L'interprétation des conséquences de ces choix sur la réception de l'œuvre par un lecteur fera appel à des considérations syntaxiques, sémantiques et stylistiques.
\end{abstract}

Keywords: introduction, direct speech, translation studies, contrastive approach, English, French, John Steinbeck, The Pearl, La perle, syntax, semantics, stylistics 
Mots-clés : introduction, discours direct, traduction, approche contrastive, anglais, français, John Steinbeck, The Pearl, La perle, syntaxe, sémantique, stylistique

Contributor: Grégoire Lacaze is Lecturer in English linguistics at Aix-Marseille Université. He wrote his $\mathrm{PhD}$ on the introduction of direct speech in present-day English. His research deals with linguistics, stylistics, semantics, and discourse analysis applied to the study of reported speech. He teaches English linguistics, grammar and translation at Aix-Marseille Université.

\section{Introduction}

Envisager une approche contrastive pour étudier l'introduction du discours direct en anglais et en français, c'est se proposer d'interroger, d'une part, les choix effectués par des auteurs anglophones, et, d'autre part, les stratégies adoptées par les traducteurs de ces œuvres qui doivent adapter leur production textuelle afin que leur traduction obéisse aux contraintes syntaxiques du français. Cette étude va tenter d'apporter quelques éclairages sur la méthodologie adoptée par les traducteurs Marcel Duhamel et Renée Vavasseur dans La perle ${ }^{1}$, traduction française de The Pearl de John Steinbeck parue aux Éditions Gallimard en 1950. L'interprétation des conséquences de ces choix sur la réception de l'œuvre par un lecteur fera appel à des considérations syntaxiques, sémantiques et stylistiques.

L'on pourrait fort bien comparer l'introduction du DD dans des œuvres de fiction en anglais et en français. Toutefois, ce n'est pas la démarche méthodologique d'analyse qui sera adoptée dans cette étude. L'œuvre en langue française étudiée ici n'a pas été créée ex nihilo, elle est l'adaptation en français d'une œuvre écrite originellement en anglais américain. Autrement dit, l'œuvre de Steinbeck préexiste avant toute tentative de traduction vers une langue autre.

Cette étude, par des va-et-vient constants entre des séquences textuelles écrites dans les deux langues, cherche à faire émerger certaines stratégies récurrentes qui ont pu guider et orienter l'activité de traduction de The Pearl. Elle tend ainsi à éclairer les choix de traduction retenus par les traducteurs français d'une œuvre de fiction en langue anglaise.

1 Pour des raisons de commodité, l'édition choisie est celle publiée aux Éditions Gallimard en 2000 dans laquelle le texte source occupe les pages paires alors que la traduction occupe les pages impaires. Ainsi, la traduction se trouve toujours en regard du texte source. 
Regards croisés sur l'introduction du discours direct en anglais et en français : mise en contexte dans The Pearl et La perle de John Steinbeck

\section{L'introduction du DD : une méthodologie d'analyse}

\section{Le segment contextualisant annonceur de DD et le discours cité}

En tant qu'énoncé de discours rapporté (noté DR), un énoncé de discours direct (noté DD) met en jeu deux niveaux énonciatifs : le report de paroles effectué par un locuteur rapporteur, d'une part, et l'acte énonciatif prétendument attribué à un locuteur origine, d'autre part. Cette remarque d'ordre général n'est pas spécifiquement rattachée à une langue donnée. Même si une approche contrastive sera privilégiée pour analyser les divers énoncés, il ne semble pas nécessaire, à ce stade de l'étude, de distinguer l'anglais et le français quant à la définition d'un énoncé de DR.

Dans la méthodologie d'analyse que nous avons élaborée pour analyser un énoncé de DD en anglais, nous avons introduit le concept de "segment contextualisant annonceur de DD», encore noté SC de DD (Lacaze 2011). Dans cette approche globalisante qui est indépendante du genre discursif auquel l'énoncé étudié appartient, il a été établi qu'un énoncé de DD associe généralement deux séquences textuelles: le SC de $\mathrm{DD}$, qui annonce les paroles d'un locuteur origine et dont le choix de composition relève pleinement de l'investissement personnel du locuteur rapporteur, et le discours cité, qui reproduit les propos qu'un locuteur origine est censé avoir tenus. Cette réflexion conceptuelle ne pose pas comme préalable l'étude d'une occurrence de DD pour un énoncé écrit en langue anglaise. Ainsi, la terminologie adoptée ici est tout à fait transposable à l'étude d'une autre langue comme le français par exemple.

Pour autant, la mention d'une dichotomie entre ces deux séquences textuelles ne doit pas laisser penser que la limite séparative entre un discours cité et le SC qui l'introduit est nécessairement bien définie. Cette zone qui sépare des fragments du discours d'un locuteur origine et des fragments du discours d'un locuteur rapporteur se caractérise parfois par une certaine plasticité, voire un certain degré de porosité entre ces séquences textuelles. C'est notamment le cas des occurrences de «DD imbriqué » dans lesquelles des fragments du discours origine sont insérés dans le discours du rapporteur avec ou sans marquage typographique. De telles occurrences de DR se rencontrent indifféremment en anglais et en français et peuvent être la trace de discours rapportés hybrides, qui sont des énoncés présentant une «mixité formelle $»^{2}$ entre plusieurs formes de DR.

2 L'expression est empruntée à Laurence Rosier $(1999,161)$. 


\section{L'importance d'une approche contextualisante}

L'approche méthodologique que nous privilégions pour étudier une occurrence de DD donnée repose sur la prise en compte de l'environnement cotextuel de cette occurrence de DD. Ainsi, l'occurrence de DD n'est pas envisagée isolément de ses cotextes gauche et droit mais elle est mise en relation avec les segments textuels qui se situent dans son voisinage. Ainsi, cette étude transphrastique peut contribuer à mettre en relief les phénomènes de cohésion et de cohérence discursives qui sont à l'œuvre dans une production textuelle. Cette approche contextualisante, qui a été introduite dans nos travaux sur l'anglais (Lacaze 2011), peut sans difficulté particulière être généralisée à d'autres langues.

\section{Le SC de DD envisagé comme un des "universaux de traduction »}

L'hypothèse que nous soutenons est que toute occurrence de DD peut s'analyser en fonction de son environnement cotextuel. Elle contient donc généralement un SC de DD. En effet, le SC a une fonction essentielle : il assure l'attribution de paroles ou de pensées à une origine énonciative. Cependant, le SC peut être effacé. Dans ce cas, le discours cité n'est plus introduit et c'est au lecteur de chercher à rétablir l'origine énonciative manquante à partir d'indices textuels mais aussi à partir de la représentation, de la mise en scène qu'il peut se faire de l'acte énonciatif origine. Pour cela, le lecteur doit tenir compte du nombre de participants dans la situation d'énonciation origine et il doit évaluer la légitimité de la prise de parole d'un de ces participants dans le «tour de parole $»^{3}$ qui n'est pas introduit. Un tel effacement de l'origine énonciative peut aller jusqu'au phénomène de «brouillage énonciatif d'un dit $»^{4}$ (Lacaze 2010, 10).

Lorsqu'il n'est pas effacé, un SC de DD permet la mise en relation entre un discours cité et une origine énonciative. Il est présent aussi bien en anglais qu'en français. Il semble donc légitime de s'interroger sur la manière avec laquelle ce segment textuel va être traité par les traducteurs d'une œuvre en langue anglaise. Le SC apparaît alors comme un des « universaux de traduction $»^{5}$ : c'est-à-dire que l'on doit en général

3 Nous empruntons l'expression tour de parole à Catherine Kerbrat-Orecchioni (1990, 159).

Les conséquences d'un effacement de l'incise ou du SC de DD qui peuvent se manifester sous la forme d'un «brouillage énonciatif » ont été analysées à partir des tours de parole apparaissant dans la nouvelle "Hills Like White Elephants" d'Ernest Hemingway (Lacaze 2010).

Cette expression est empruntée à Mathieu Guidère $(2010,93)$ qui en donne la définition suivante : « [Les universaux de traduction] renvoient à des régularités générales de structure qui peuvent être 
Regards croisés sur l'introduction du discours direct en anglais et en français : mise en contexte dans The Pearl et La perle de John Steinbeck

retrouver sa présence dans un énoncé de DD, pour que celui-ci demeure intelligible pour un lecteur.

\section{La traduction d'une ouvre: une production textuelle sous contraintes}

\section{Différentes stratégies privilégiées par les traducteurs}

Lorsque l'on évoque la problématique de la traduction d'une œuvre de fiction, il est souvent fait mention de l'existence d'une dichotomie entre une stratégie «sourcière» et une stratégie «cibliste ${ }^{6}$ dans les études portant sur la traductologie ${ }^{7}$ (Guidère 2010, 98). Lorsqu'il entreprend la traduction d'une œuvre, un traducteur est amené à effectuer des choix. Il jouit ainsi d'un ensemble de contraintes et de libertés qui vont guider sa stratégie de traduction, comme l'indique Mathieu Guidère (2010, 12-3) :

S'il est acquis aujourd'hui que l'étude traductologique est de nature descriptive et non pas prescriptive, cela ne signifie pas que l'activité de traduction telle qu'elle s'exerce n'est pas soumise à des règles et à des normes. Celles-ci peuvent être consciemment respectées ou inconsciemment intériorisées, mais elles existent toujours en fonction des contextes et des finalités de la traduction.

Le traducteur peut chercher à privilégier une approche mimétique entre sa traduction et le texte source, il aura ainsi tendance à adopter une stratégie sourcière. Au contraire, s'il favorise la réception de l'œuvre traduite par un lectorat francophone, c'est vers une stratégie cibliste qu'il tendra.

rapportées à des contraintes articulatoires et/ou des contraintes cognitives. En traductologie, les "universaux" désignent les traits linguistiques qui apparaissent essentiellement dans les textes traduits et qui semblent indépendants des paires de langues en présence. En d'autres termes, il s'agit de caractéristiques que l'on retrouve dans les traductions quelle que soit la langue considérée. »

6 Mathieu Guidère $(2010,33)$ évoque cette « controverse entre "sourciers" et "ciblistes" : les premiers concentrent leurs efforts sur le texte source, les seconds s'intéressent davantage aux attentes du public cible».

7 Dans cette étude, nous retenons la définition du terme traductologie proposée par Mathieu Guidère $(2010,14)$ :

«La traductologie a pour objet la traduction envisagée en elle-même (processus) et pour elle-même (produit). Par "traduction", il faut donc comprendre la suite ordonnée d'opérations ayant un tenant (le texte de départ, texte source ou texte à traduire), un aboutissant (le texte d'arrivée, texte cible, texte traduit), et un acteur central (le traducteur, adaptateur, médiateur). » 
Le traducteur est ainsi envisagé comme un «médiateur» entre la production textuelle origine et la production textuelle dans une langue autre :

Le traducteur a été considéré tour à tour comme un «translateur» chargé de la simple transposition des mots d'une langue à l'autre, comme un « adaptateur » ayant la responsabilité de satisfaire les attentes du public visé, comme un «médiateur» qui se place à mi-chemin entre deux cultures ou deux mondes pour les rapprocher, comme un «communicateur » enfin, chargé de faciliter le dialogue entre individus ou communautés éloignées. (Guidère 2010, 15)

\section{Une pluralité de traductions possibles}

À partir d'un texte donné en langue anglaise, il n'existe pas une seule traduction correcte, mais l'on peut envisager une pluralité de traductions. Notre étude s'attache ici à éclairer les choix faits par les traducteurs de The Pearl pour créer une traduction en langue française de cette œuvre. Ces choix de traduction sont nécessairement influencés par le fait que les traducteurs ont une appréhension globale de l'œuvre à traduire.

Citons à ce propos Mathieu Guidère $(2010,31)$ :

[le texte à traduire] est en réalité un tout qui doit être appréhendé dans sa relation à un contexte particulier et en fonction d'une finalité précise. Si le cadre général de la traduction est bien posé, la fidélité cesse d'être un problème : elle devient un choix parmi d'autres sur la gamme des actions conscientes du traducteur.

L'idée même de fidélité au texte origine est réfutée par Henri Meschonnic $(2007,130)$ car cette fidélité impliquerait de proposer une traduction à partir de laquelle des correspondances terme à terme pourraient être établies entre le texte source et le texte cible. Il ne s'agit pas de chercher à traduire des unités linguistiques souvent réduites à des mots mais plutôt de chercher à traduire le rythme : «Parce que de toute façon le mot n'est pas l'unité du discours, mais une unité de la langue. On croit être fidèle au texte. On est fidèle au signe ». 
Regards croisés sur l'introduction du discours direct en anglais et en français : mise en contexte dans The Pearl et La perle de John Steinbeck

\section{Cohérence et cohésion dans la composition des SC}

Une traduction, en tant que production textuelle dérivée d'un texte source, est nécessairement mise en contexte avec le texte source. Dans cette étude, notre intérêt se porte sur la composition des SC. Pour un SC donné, il semble nécessairement exister certaines tensions entre un environnement cotextuel immédiat, que l'on pourrait qualifier de «contexte interne » et une mise en relation avec le texte source, qui constituerait le «contexte externe». Ces tensions sont donc génératrices de contraintes pour le traducteur. Par ailleurs, plusieurs niveaux de cohésion et de cohérence sont à l'œuvre dans un texte, comme le rappelle Mathieu Guidère $(2010,96)$ qui distingue le «niveau microtextuel (des unités lexicales et syntaxiques)» et le «niveau macrotextuel». Ainsi, le traducteur «doit penser la résolution des problèmes au niveau microtextuel en fonction de sa stratégie au niveau macrotextuel ».

Cette oscillation entre le niveau microtextuel et le niveau macrotextuel peut être mise en parallèle avec la problématique de la traduction soumise aux tensions entre le «discontinu» (le mot isolé, le signe) et le «continu» (le rythme, la prosodie), un problème décrit par Henri Meschonnic $(2007,38-9)$ pour la traduction de la poésie. Il s'agit alors de privilégier la traduction du rythme plutôt que la traduction du signe : « Donc si on ne traduit pas le rythme, on n'a pas traduit le texte, on a traduit du signe » (Meschonnic 2007, 105).

Le traducteur doit donc rechercher un compromis entre des contraintes intra-phrastiques et des contraintes inter-phrastiques. Tel semble être le défi également posé pour la traduction des segments d'introduction du DD. Geneviève Salvan (2005 114), qui a étudié l'incise de discours rapporté dans le roman français, précise bien l'enjeu de l'incise, ou par extension du SC de DD, dans ce jeu de tensions entre « cohésion interne » et « cohérence englobante »:

Nous pensons que le rôle de l'incise est passé globalement d'un principe de cohésion interne (fonction au sein de la phrase), de lisibilité locale (signalement des tours de parole, identification et désignation du locuteur, voire caractérisation de l'acte de langage) à celui de cohérence englobante (passage récit/discours, commentaires métadiscursifs, stratégies narratoriales).

Cette tension va avoir des répercussions concrètes sur la composition des SC de DD, comme nous allons le voir. 


\section{L'introduction du discours direct dans The Pearl et La perle}

\section{Le discours direct dans The Pearl}

De nombreuses occurrences de dialogue apparaissent dans cette novella. Pour autant, ces dialogues ne contiennent parfois qu'un nombre limité de répliques et les répliques de différents personnages peuvent être séparées par des séquences de récit alors que ces répliques tendraient à s'enchaîner les unes aux autres si elles étaient prononcées dans le monde extralinguistique. Ces occurrences de DD sont ainsi mises en relief par les choix énonciatifs du narrateur. L'on peut aussi faire la remarque suivante : alors qu'un dialogue pourrait permettre de faire résonner les voix des personnages, le narrateur préfère souvent évoquer la présence sous-jacente et récurrente de diverses mélodies qui jouent un rôle primordial dans la diégèse, comme l'évoque Linda Wagner-Martin (1994, xx) dans son introduction à The Pearl :

To replace verbal meaning, Steinbeck creates a technique suitable for a film script but unexpected in a written text. He uses music both to express mood and to replace dialogue. His "Song of the Family," a positive and encouraging sound, is set against the "Song of Evil" or the "Song of the Pearl." What happens in the struggles among the refrains anticipates the narrative conflicts.

\section{Influence de la chaîne référentielle sur la composition d'un SC de DD}

Lorsqu'un SC est présent pour introduire une occurrence de DD, le choix du sujet syntaxique qui désigne la source énonciative est fortement influencé par l'environnement cotextuel d'une occurrence de DD donnée. L'énoncé suivant illustre cette idée :

Kino nodded, and only then did she let the doctor take the baby.

"Hold the light," the doctor said, and when the servant held the lantern high, the doctor looked for a moment at the wound on the baby's shoulder. ${ }^{8}$ (Steinbeck 96)

Kino approuva de la tête et seulement alors permit-elle au docteur de prendre l'enfant.

8 Sauf indication contraire, tous les gras figurant dans les énoncés étudiés sont de nous. 
Regards croisés sur l'introduction du discours direct en anglais et en français : mise en contexte dans The Pearl et La perle de John Steinbeck

«Tenez la lumière », dit-il, et quand le domestique eut levé la lanterne, il considéra la blessure à l'épaule du petit. (97)

Dans le texte source, le SC de DD contient le syntagme défini the doctor. En raison de la présence de plusieurs participants à l'acte énonciatif, Kino, sa femme Juana, son fils Coyotito, le médecin et le domestique, la source énonciative doit être précisée pour lever toute ambiguïté. L'emploi $\mathrm{du}$ pronom personnel he à la place du syntagme nominal the doctor ne permettrait pas d'identifier aisément qui parle car plusieurs personnages masculins sont présents dans l'univers diégétique.

Dans la traduction, le choix fait par les traducteurs conduit à la mention du pronom endo-anaphorique il. Pour identifier la source énonciative du dit qui est rapporté, il faut parcourir à rebours le cotexte gauche à la recherche de la mention d'un participant masculin à l'acte énonciatif origine. Il s'agit alors de parcourir la chaîne référentielle qui a abouti à la désignation du locuteur origine par un pronom personnel. Le très jeune enfant n'étant pas en mesure d'être un locuteur origine, c'est bien le docteur qui est à l'origine du contenu du discours cité. Ayant pris l'enfant dans ses bras, sa prise de parole est légitime. Par ailleurs, la présence dans le cotexte droit d'une description de l'activité du domestique au service du médecin tend à confirmer l'identité du locuteur désigné par le pronom personnel $i l$. Pour autant, Kino aurait pu être envisagé également comme une source énonciative potentielle. L'analyse du contenu du discours cité ainsi que la prise en compte du cotexte droit du discours cité incitent à attribuer le tour de parole au docteur. Dans cette occurrence de DD, les conditions $\mathrm{du}$ 《réalisme langagier » et du « réalisme pragmatique ${ }^{9}$ sont assurées: le docteur est bien le locuteur origine dont les propos sont rapportés dans cet énoncé de DD.

Les contraintes au niveau macrotextuel ont également une influence sur la composition des séquences au niveau microtextuel : into Kino.

"He would not come," the people in the yard said.

"He would not come," the people in the door said, and the thought got

“The doctor would not come," Kino said to Juana. (Steinbeck 38)

«Il ne voudra pas venir », dirent les gens dans la cour.

9 Les concepts de «réalisme langagier» et de « réalisme pragmatique » ont été introduits par Monique De Mattia-Viviès $(2006,110)$ pour identifier une occurrence de discours indirect libre. Nous adaptons ces concepts pour une autre forme de discours rapporté : le DD. 
«Il ne voudra pas venir», dirent les gens dans la porte, et Kino se laissa envahir par cette pensée.

« Le docteur ne voudra pas venir », dit-il à Juana. (39)

L'ordre canonique sujet-verbe se retrouve dans les incises des trois répliques en anglais. Dans la traduction, les trois répliques contiennent une inversion sujet-verbe d'incise. Dans la troisième réplique, il semble que la mention du complément d'objet indirect à Juana ait conduit à la substitution de l'appellatif Kino par le pronom personnel il. Au niveau microtextuel, l'incise dit-il à Juana a pu être choisie en lieu et place de l'incise dit Kino à Juana car l'identité du locuteur est présente dans le cotexte gauche du discours cité.

L'analyse transphrastique qui a été menée sur l'énoncé ci-dessus illustre l'hypothèse selon laquelle une occurrence de DD n'est pas traduite indépendamment de son environnement cotextuel. C'est la mise en contexte de cette occurrence qui permet aux traducteurs de proposer une traduction respectant cohérence et cohésion discursives.

\section{Inversion sujet-verbe dans le SC de DD}

Cette inversion est quasi-obligatoire à l'écrit en français pour assurer la grammaticalité de l'énoncé. Il en va autrement en anglais, comme l'analyse Lucie Gournay $(2000,36)$ qui compare l'ordre des termes dans les incises en français et en anglais :

En français, ces énoncés sont traditionnellement agencés selon le schéma Verbe - Sujet - (compl). [...]

L'inversion Verbe-Sujet dans ces énoncés français en incise semble faire partie du code de la narration, et ne subit pas de variante, quel que soit le type de sujet [...], ou quel que soit le verbe [...].

En anglais, les énoncés en incise sont par rapport au français structurellement plus variés puisque l'on trouve 2 schémas en apparente concurrence : Verbe - Sujet - (compl) et Sujet - Verbe - (compl).

Si l'on s'intéresse à la distribution de l'ordre sujet-verbe d'incise dans The Pearl, l'on peut constater que le narrateur-locuteur rapporteur tend plutôt à privilégier l'ordre canonique sujet-verbe, indépendamment de la composition du SC. Les occurrences de répliques dans lesquelles apparaît une inversion sujet-verbe sont relativement marginales: seules 28 occurrences sont recensées. Sur ces 28 occurrences, aucune ne contient un pronom personnel en position de syntagme nominal sujet en collocation avec un verbe introducteur de parole. Cette remarque semble être en accord 
Regards croisés sur l'introduction du discours direct en anglais et en français : mise en contexte dans The Pearl et La perle de John Steinbeck

avec les travaux de Lucie Gournay $(2000,39)$ qui émet l'hypothèse suivante :

Si l'énoncé du type said he n'est pour ainsi dire pas attesté dans les textes contemporains, ce n'est pas parce qu'il existe une contrainte syntaxique liée à la nature pronominale du sujet. C'est plutôt parce qu'il existe une forte contrainte énonciative qui s'est développée récemment dans les textes de fiction.

Dans son corpus, la linguiste relève un unique emploi dans lequel un pronom personnel suit le verbe d'incise, ce qu'elle interprète comme « un discours direct dont le contenu peut être perçu comme archaïque » sans pour autant rejeter la grammaticalité d'une telle construction. Elle constate plutôt qu'il s'agit là de «l'expression d'un archaïsme de convention littéraire » (2000, 39).

Par ailleurs, le choix d'emploi du verbe say comme verbe de parole ne semble pas influer sur l'ordre sujet-verbe dans The Pearl, le verbe say étant le plus souvent employé dans des incises respectant l'ordre canonique sujet-verbe. De plus, sur les 28 occurrences d'inversion sujet-verbe, une seule contient le verbe ask, toutes les autres contenant le verbe prototypique du report de paroles, le verbe say.

L'on peut mentionner que 18 occurrences d'inversion apparaissent dans des extraits de dialogue entre Kino et son frère Juan Tomás, qui est un personnage bien moins présent que Juana, la femme de Kino. Dans les quelques échanges énonciatifs rapportés entre Kino et son frère, l'inversion sujet-verbe est très fréquente. Elle se justifie peut-être par la longueur du syntagme nominal sujet qui contient le nom composé Juan Tomás, comme dans l'extrait suivant :

"To the north," said Kino. "I have heard that there are cities in the north."

"Avoid the shore," said Juan Tomás. [...]

"I have it," said Kino.

$[\ldots]$

"The wind is good," said Juan Tomás. (Steinbeck 180)

Par souci d'harmonie, l'inversion sujet-verbe dans une réplique de Juan Tomás entraînerait une inversion dans les répliques contiguës attribuées à son frère Kino. 


\section{Différences entre le texte source et sa traduction et répercussions sémantiques des choix des traducteurs}

Des différences syntaxiques notables voient le jour entre le texte source et le texte cible, en ce qui concerne les structures d'introduction du DD. Ces changements peuvent affecter la syntaxe de la phrase à des degrés divers. Ils peuvent également impliquer une altération de la ponctuation entre l'énoncé en langue anglaise et l'énoncé traduit, ce qui apparaît souvent comme l'élément le plus visible. Cet article envisagera seulement les différences de ponctuation matérialisant la frontière entre SC et discours cité.

\section{Différences de ponctuation entre le SC de DD et le discours cité}

\section{Normes éditoriales et typographiques}

Les normes éditoriales et typographiques étant différentes en anglais et en français, il n'est pas étonnant de retrouver ces différences dans The Pearl et sa traduction. Ainsi, les double quotes présents dans The Pearl sont remplacés par les guillemets français en forme de doubles chevrons. En anglais, les guillemets encadrent chaque fragment de discours cité. Dans une occurrence de DD, le SC n'est pas délimité par des guillemets. Il est donc mis en évidence visuellement comme n'appartenant pas au discours cité. En français, les guillemets encadrent la réplique ${ }^{10}$ d'un personnage. Visuellement, il est ainsi moins aisé de repérer le SC en français car il est en quelque sorte «noyé » dans les paroles origines du personnage contrairement à l'anglais. Cette remarque semble réservée à la fiction romanesque en français. En effet, d'autres normes typographiques sont utilisées en discours journalistique. Par exemple, dans le quotidien français Le Monde, chaque occurrence de DD est délimitée par des guillemets. Dans cette séquence textuelle entre guillemets, le discours cité est en italiques, ce qui le démarque visuellement du SC qui l'introduit.

Les guillemets anglais, lorsqu'ils sont utilisés dans la fiction pour rapporter un $\mathrm{DD}$, contribuent à isoler de manière nette les fragments textuels pris en charge par un locuteur origine. Ainsi, les ruptures typographiques matérialisées par les guillemets coïncident généralement avec les frontières

10 Par réplique, nous entendons à la fois le discours cité et le SC annonceur de DD, lorsqu'il est présent. 
Regards croisés sur l'introduction du discours direct en anglais et en français : mise en contexte dans The Pearl et La perle de John Steinbeck

entre le discours cité et le SC qui l'introduit. Il en va autrement en français, en particulier lorsqu'un SC se retrouve en position médiane relativement au discours cité : le SC est alors encadré par deux fragments de discours cité et la frontière entre le $\mathrm{SC}$ et un fragment de discours cité est souvent matérialisée par une virgule. Hélène Chuquet et Michel Paillard (1987, 419), qui comparent la ponctuation en anglais et en français, relèvent qu'en anglais, «les verbes introducteurs ne sont pas inclus dans les guillemets, qu'ils soient ou non en incise», tandis qu'en français, «les incises ne s'isolent pas des parties entre guillemets et restent incluses dans la citation ». De manière analogue, Denis Keen et Valérie Binet $(2009,134)$ font la remarque suivante: «Dans un dialogue en français, les incises ne s'isolent pas des parties entre guillemets et restent incluses dans la citation. Ce n'est pas le cas en anglais $» 11$.

\section{Porosité entre le SC et le discours cité}

Les traducteurs de The Pearl ont, semble-t-il, cherché le plus souvent à assurer une meilleure intégration du report de paroles dans la trame narrative. La frontière entre le récit et une occurrence de DD devient ainsi souvent plus poreuse dans le texte français sans que cette tendance soit une constante et apparaisse comme une règle à laquelle les traducteurs se refusent à déroger.

En anglais, nous avons montré qu'un SC peut être syntaxiquement indépendant du discours cité qu'il annonce (Lacaze 2011). Dans le cas d'un $\mathrm{SC}$ en position initiale, cette rupture peut être représentée par un point entre deux phrases autonomes. En français, il arrive que cette frontière entre SC et discours citant soit matérialisée par l'emploi des deux-points au niveau de la zone de contact entre SC et discours cité. Ceci est illustré dans l'extrait suivant de The Pearl et dans sa traduction en français :

The man who looked out at him was one of his own race. Kino spoke to him in the old language. "The little one - the first-born - has been poisoned by the scorpion," Kino said. "He requires the skill of the healer." (Steinbeck 44)

L'homme qui l'observait de l'intérieur était de sa race. Kino lui parla dans leur langue natale : «Le petit... notre premier-né... a été empoisonné par le scorpion, expliqua-t-il. Il a besoin de la science du guérisseur. » (45)

11 Ce sont les auteurs qui soulignent le terme incises. 
Dans l'énoncé en français, la frontière entre le SC et le discours cité tend à s'estomper et le discours cité est davantage imbriqué dans la trame narrative.

Dans la traduction, l'annonce du report d'un acte de parole est souvent anticipée dans la phrase qui précède le report effectif. Cette phrase du cotexte gauche joue davantage encore le rôle de segment contextualisant car il réduit le champ des possibles et oriente le lecteur vers la retranscription du report de paroles qui suit, comme le montre l'extrait suivant :

And now she did a most surprising thing.

"The doctor," she said. "Go to get the doctor."

The word was passed out among the neighbours where they stood close-packed in the little yard behind the brush fence. (Steinbeck 36)

Et, soudain, elle eut le mot le plus inattendu :

«Le docteur, dit-elle. Va chercher le docteur!»

De bouche en bouche, le mot passa parmi les voisins massés dans la petite cour derrière la haie. (37)

Alors que l'action de Juana n'est pas précisée avant sa prise de parole dans le texte source, il ne subsiste aucune ambiguïté sur son activité de nature langagière dans la traduction. Par ailleurs, le syntagme nominal le mot est mentionné avant que les paroles prononcées soient rapportées. Ce syntagme nominal est ensuite repris dans le cotexte droit, ce qui aboutit à un effet de répétition qui n'existait pas dans le texte source. Il y a donc une accentuation de l'effet de répétition qui insiste sur la «circulation » $12 \mathrm{du}$ discours origine. Par ailleurs, des changements syntaxiques existent entre le texte source et sa traduction car le report de paroles est précédé des deuxpoints en français, ce qui tend à intégrer davantage le report de paroles dans la trame narrative.

Pourtant, le changement syntaxique lié au changement de ponctuation n'est pas automatique dans la traduction. La structure d'un SC autonome syntaxiquement du discours cité peut être conservée dans la traduction en français. Ainsi, l'occurrence de DD n'est pas introduite au sens strict par une incise. L'attribution énonciative d'une occurrence de discours cité peut se faire par simple contiguité textuelle entre le SC autonome et le discours cité qui le suit :

12 Ce terme est emprunté à Laurence Rosier $(2008,132)$. 
Regards croisés sur l'introduction du discours direct en anglais et en français : mise en contexte dans The Pearl et La perle de John Steinbeck

The doctor looked surprised. "I had not heard of it. Do you keep this pearl in a safe place? Perhaps you would like me to put it in my safe?" (Steinbeck 106)

Le docteur eut l'air surpris. «Je n'en ai rien entendu. L'avez-vous mise en sécurité ? Peut-être voudriez-vous que la dépose dans mon coffre ?» (107)

Les propositions The doctor looked surprised et Le docteur eut l'air surpris sont syntaxiquement indépendantes des discours cités qu'elles introduisent au niveau sémantique. Ainsi, pour le texte source comme pour la traduction, «il existe une subordination sémantique entre le segment introducteur et le discours cité qui suit » (Lacaze 2011,34). Même en l'absence de verbe de parole, le SC en position initiale assure la fonction d'attribution d'un dit à une origine énonciative grâce à la mention du syntagme défini the doctor qui désigne la source énonciative des paroles qui sont rapportées ensuite.

Dans The Pearl, la présence d'une occurrence de «discours narrativisé ${ }^{13}$ (noté $\mathrm{DN}$ ) peut être concomitante à la présence des deuxpoints séparant le récit d'un report de paroles au DD, comme l'illustre l'extrait suivant :

Kino held his breath, and the neighbours held their breath, and the whispering went back through the crowd: "He is inspecting it - No price has been mentioned yet - They have not come to a price." (Steinbeck 138)

Alors que l'insertion d'un DN dans le texte source tend à atténuer la séparation entre récit et DD dans le texte source, cette séparation est plus marquée dans la traduction par l'indépendance syntaxique entre le SC et le discours cité qui le suit :

Kino, les voisins, tous retinrent leur souffle et un chuchotement passa dans la foule. «Il est en train de l'examiner... On n'a pas encore fait mention du prix... Ils n'ont pas encore convenu du prix. » (139)

Compte tenu des choix retenus par les traducteurs qui n'adoptent pas toujours le même signe de ponctuation pour un SC en position initiale, il

13 L'expression discours narrativisé est empruntée à Gérard Genette $(1972,191)$ qui la définit ainsi : «un discours "narrativisé", c'est-à-dire traité comme un événement parmi d'autres et assumé comme tel par le narrateur lui-même ». 
semble envisageable de parler de ponctuation «fluctuante» pour caractériser la frontière poreuse entre le $\mathrm{SC}$ et le discours cité qui le suit.

Ponctuation «fluctuante » entre le SC et le discours cité

Dans le texte source, trois signes de ponctuation entrent en concurrence pour conclure les SC en position initiale : la virgule, les deuxpoints et le point. Dans la traduction, la seule alternative possible aux deuxpoints est la présence d'un point qui entraîne l'indépendance syntaxique entre $\mathrm{SC}$ et discours cité.

La virgule est utilisée dans les deux énoncés suivants :

And Juan Tomás, who squatted on Kino's right hand because he was his brother, asked, "What will you do now that you have become a rich man?" (Steinbeck 78)

All of these things Kino saw in the lucent pearl, and he said, "We will have new clothes." (Steinbeck 80)

La virgule crée une rupture au niveau syntaxique mais à un degré moindre que les deux-points, comme en témoigne l'analyse de Randolph Quirk et al. $(2005,1620)$ :

The colon makes a sharper separation than the comma $[\ldots]$ it indicates a closer interdependence between the units separated than does the semicolon. Indeed, it sometimes indicates as close a relation as the comma does, but it is a different relation.

La virgule apparaît alors comme une marque de ponctuation bien plus ténue que les deux-points. La grammaire citée ci-dessus souligne la fonction de mise en relation par juxtaposition qui caractérise l'utilisation d'une virgule dans une phrase: "The comma is the most flexible of all the punctuation marks [...]. One dominant use of the comma is to separate closely associated clauses within a sentence » $(2005,1615)$. Pour JeanRémi Lapaire et Wilfrid Rotgé (1991, 555), «l'emploi de la virgule entre plusieurs propositions suppose une relation sémantique étroite entre elles [...]. »14. Les deux linguistes résument ainsi la double fonction de la virgule : «flexibilité et séparation de ce qui est étroitement associé » (556).

14 Ce sont les auteurs qui soulignent l'expression. 
Regards croisés sur l'introduction du discours direct en anglais et en français : mise en contexte dans The Pearl et La perle de John Steinbeck

Lorsque les deux-points sont utilisés après un SC en position initiale, la rupture est bien plus nette avec le discours cité. En cela, les deux-points peuvent être assimilés à une frontière marquée délimitant le discours citant et le discours cité, comme dans l'extrait suivant :

In the pearl he saw Juana and Coyotito and himself standing and kneeling at the high altar, and they were being married now that they could pay. He spoke softly: "We will be married — in the church." (Steinbeck 80)

En étant tout à la fois un constituant de la phrase contenant le SC mais un constituant séparé du segment introducteur par les deux-points, le discours cité attire l'attention du lecteur car il occupe une position rhématique dans cet énoncé. Par ailleurs, l'acte de parole est lui aussi mis en relief comme si la prise de parole de Kino acquérait la valeur d'un acte fondateur. Ce faisant, l'attention du lecteur est alors mobilisée pour qu'il lise attentivement le contenu du discours cité qui suit.

\section{Conclusion}

Cette étude a tenté de mettre en évidence certaines stratégies mises en œuvre par les traducteurs français de La perle pour introduire du discours direct. Ces stratégies ont pu être mises au jour grâce à l'analyse de certains choix retenus par les traducteurs. À travers la variation de composition d'une chaîne référentielle pour un locuteur donné, l'inversion sujet-verbe dans un SC ou le choix d'une ponctuation différente entre le texte source et sa traduction en français, les traducteurs de The Pearl ont, semble-t-il, cherché le plus souvent à proposer aux lecteurs francophones un texte privilégiant la fluidité de lecture même si cela impose parfois de remanier en conséquence l'enchaînement des termes. Ces modifications entre le texte source et sa traduction changent ainsi notablement la réception de ces deux textes respectivement par un lecteur anglophone et par un lecteur francophone. En procédant ainsi, les traducteurs tendent à accorder davantage d'importance au rythme qu'au signe, en adoptant une approche théorique de la traduction proche de celle prônée par Henri Meschonnic (2007) :

Autrement dit, plus que ce qu'un texte dit, c'est ce qu'il fait qui est à traduire ; plus que le sens, c'est la force, l'affect.

Ce n'est donc plus de la langue qui est à traduire mais un système de discours, pas le discontinu mais le continu. (55) 
Ce conflit, c'est celui du rythme et du signe. C'est celui du continu et du discontinu [...]. (44)

En comparant le texte source et sa traduction en français, nous avons montré que les changements syntaxiques se font de proche en proche mais que les SC co-existent généralement dans le texte source et dans le texte cible car ils sont des éléments essentiels à la compréhension du déroulement de l'échange interlocutif rapporté par le locuteur rapporteur.

Ainsi, la méthodologie suivie par les traducteurs pour l'introduction du discours direct dans La perle semble être la suivante : identifier les SC annonceurs de DD dans le texte source et créer une production textuelle dans laquelle les SC permettent au lecteur d'identifier le locuteur origine et contiennent, le cas échéant, des informations sur les conditions de réalisation de l'acte de parole lorsque celles-ci sont présentes dans le texte source, comme la manière de dire, par exemple. Pour y parvenir, les traducteurs n'hésitent pas à proposer des changements syntaxiques plus ou moins radicaux si ceux-ci permettent de garantir la fluidité et le rythme de la production textuelle qu'ils créent.

La traduction proposée par les traducteurs constitue donc une production textuelle sous contraintes. Afin de mener à bien cette opération de traduction, les traducteurs peuvent notamment jouer avec la ponctuation pour privilégier la réception du texte par un lecteur francophone. Les différences entre le texte source et sa traduction peuvent être plus ou moins importantes du moment que la fonction d'attribution d'un dit à une origine énonciative demeure dans la traduction. Par sa fonction centrale d'introduction du DD, le SC est un segment textuel qui est modelé, façonné par les traducteurs pour qu'il réponde à leurs attentes. C'est pourquoi il a autant retenu notre attention dans cette analyse contrastive anglais-français.

\section{Bibliographie}

\section{Corpus littéraire}

\section{A. Euvres de langue anglaise}

STEINBECK, John, (1947) 2000. The Pearl, Paris, Gallimard. , (1947) 1994. The Pearl, London, Penguin. 
Regards croisés sur l'introduction du discours direct en anglais et en français : mise en contexte dans The Pearl et La perle de John Steinbeck

\section{B. Traduction}

DUHAMEL, Marcel et VAVASSEUR, Renée, (1950) 2000. Trad., La perle, Paris, Gallimard.

\section{Autres ouvrages cités}

CHUQUET, Hélène et PAILLARD, Michel, 1987. Approche linguistique des problèmes de traduction - Anglais-français, Paris, Ophrys.

DE MATTIA-VIVIÈS, Monique, 2006. Le discours indirect libre au risque de la grammaire: le cas de l'anglais, Aix-en-Provence, Publications de l'Université de Provence.

GENETTE, Gérard, 1972. Figures III, Paris, Seuil.

GUIDÈRE, Mathieu, (2008) 2010. Introduction à la traductologie - Penser la traduction : hier, aujourd'hui, demain, Bruxelles, De Boeck Université.

GOURNAY, Lucie, 2000 (33-64). «Linguistique contrastive et narratologie : dit-il, he said... Ce que nous apprend l'analyse linguistique des énoncés en incise dans le discours direct sur la relation entre récit et discours direct », Linguistique contrastive et traduction 5, Jacqueline Guillemin-Fischer (éd), Gap, Ophrys.

JAUBERT, Anna, 2005 (7-12). «Introduction. Cohésion et cohérence : étapes et relais pour l'interprétation », Cohésion et cohérence: études de linguistique textuelle, Anna Jaubert (éd), Lyon, ENS Éditions.

KEEN, Denis et BINET, Valérie, 2009. Le thème anglais : littérature et presse, Paris, Ophrys.

KERBRAT-ORECCHIONI, Catherine, 1990. Les interactions verbales. Tome I, Paris, Armand Colin.

LACAZE, Grégoire, 2011 (25-44). « De l'incise au segment contextualisant : un changement d'horizon dans l'introduction du discours direct », Études de Stylistique Anglaise $\mathrm{N}^{\circ} 1$.

2010. «Une analyse d'une production de discours rapporté : l'introduction des tours de parole dans la nouvelle "Hills Like White Elephants" d'Ernest Hemingway », E-rea 8.1. [http://erea.revues.org/pdf/1348]

LAPAIRE, Jean-Rémi et ROTGÉ, Wilfrid, 1991. Linguistique et grammaire de l'anglais, Toulouse, Presses Universitaires du Mirail. 
MESCHONNIC, Henri, 2007. Éthique et politique du traduire, Lagrasse, Verdier, 2007.

QUIRK, Randolph, GREENBAUM, Sidney, LEECH, Geoffrey and SVARTVIK, Jan, 2005. A Comprehensive Grammar of the English Language, London, Longman.

ROSIER, Laurence, 1999. Le discours rapporté: histoire, théories, pratiques, Bruxelles, Duculot.

--------, 2008. Le discours rapporté en français, Paris, Ophrys.

SALVAN, Geneviève, 2005 (113-144). "L'incise de discours rapporté dans le roman français du $\mathrm{XVIII}^{\mathrm{e}}$ au $\mathrm{XX}^{\mathrm{e}}$ siècle: contraintes syntaxiques et vocation textuelle», Cohésion et cohérence: études de linguistique textuelle, Anna Jaubert (éd), Collection Langages, Lyon, ENS Éditions.

WAGNER-MARTIN, Linda, 1994 (vii-xxiv). Introduction, The Pearl, John Steinbeck, London, Penguin. 